\title{
Short communication: Evaluation of the efficacy of novel disbudding methods for dairy calves
}

\author{
M. A. Sutherland, ${ }^{1 *}$ F. J. Huddart, ${ }^{1}$ and M. Stewart ${ }^{2}$ \\ ${ }^{1}$ AgResearch Ltd., Ruakura Research Centre, Hamilton 3214, New Zealand \\ ${ }^{2}$ Greyhound Racing New Zealand, TAB Building, Lower Hutt 5012, Wellington, New Zealand
}

\section{ABSTRACT}

The objectives of this study were to (1) evaluate whether cryoablation or the administration of clove oil was as efficacious as cautery disbudding at preventing horn growth, and (2) evaluate whether the efficacy of cautery disbudding is affected by removing or leaving the horn bud tissue intact after disbudding of dairy calves. At approximately $4 \mathrm{~d}$ of age $(4.0 \pm 0.88 \mathrm{~d}$ of age, mean $\pm \mathrm{SD}), 265$ dairy heifer calves from 3 dairy farms (farm 1: $\mathrm{n}=129$ calves; farm 2: $\mathrm{n}=109$ calves; farm $3: \mathrm{n}=27$ calves) were disbudded. Each calf had 1 of 4 treatments randomly assigned to each horn bud: (1) clove oil $(0.5 \mathrm{~mL})$ administered subcutaneously under the horn bud (CLOV, $\mathrm{n}=135$ buds); (2) a liquid nitrogen-filled probe applied to the horn bud area for 30 $\mathrm{s}$ (CRYO, $\mathrm{n}=134$ buds); (3) cautery disbudding using an electric hot-iron and the horn bud removed (BUDOFF, $\mathrm{n}=130$ ); or (4) cautery disbudding and the horn bud tissue left intact (BUDON, $\mathrm{n}=131$ ). Calves were assessed for signs of infection at the disbudding site frequently within the first month after disbudding. At approximately 6 mo of age $(6 \pm 2.2$ mo, mean \pm $\mathrm{SD})$ calves were assessed for scur or horn growth. The disbudding procedures were considered successful if no scur or horn development was observed. Within the first month, $12 \%$ of disbudding wounds showed some indication of infection, such as pus, exudate, or swelling; of the infected buds, $52 \%$ were associated with the BUDON treatment, $27 \%$ with CLOV, $25 \%$ with BUDOFF, and $2 \%$ with CRYO treatments. At 6 mo of age, BUDOFF was the most effective method of preventing horn growth and CRYO was the least efficacious [mean percentage of success: BUDOFF: 100\% (95\% CI: 97.7-100.0); CRYO: 1\% (95\% CI: 0.2-5.3)]. Injecting clove oil under the horn bud was $87 \%$ (95\% CI: 80.6-92.5) successful. Not removing the horn bud

Received June 17, 2018.

Accepted September 20, 2018.

*Corresponding author: mhairi.sutherland@agresearch.co.nz tissue after cautery disbudding reduced the efficacy of this method by $9 \%$ [91\% success (95\% CI: 83.8-95.7)]; moreover, this method was associated with more infection at the site of disbudding. It appears as though the clove oil treatment could be used as an alternative to cautery disbudding of dairy calves; however, further research is needed to evaluate the tissue damage and associated pain caused by clove oil and to refine this technique (i.e., administration methods to improve efficacy) before it could be considered an alternative to cautery.

Key words: calf, cautery, clove oil, cryoablation, horns

\section{Short Communication}

Dairy calves are commonly disbudded to prevent horn growth because horns can cause injury to other animals and stock people. Disbudding is often performed using a heated cautery iron (Stafford and Mellor, 2011; Cozzi et al., 2015; USDA, 2018). This procedure is known to cause behavioral and physiological changes indicative of pain if no pain relief is given (reviewed by Stafford and Mellor, 2011). Other methods used to prevent horn growth, such as applying caustic paste to the horn bud (Morisse et al., 1995; Stilwell et al., 2009) and amputation dehorning (McMeekan et al., 1998; Sylvester et al., 2004), have also been reported to cause behavioral and physiological changes indicative of pain if no pain relief is given. Providing calves with pain relief, such as a local anesthetic block and nonsteroidal anti-inflammatory drugs, can eliminate the behavioral and physiological response to cautery disbudding (Stafford and Mellor, 2011). Moreover, selecting for polled dairy cattle would ultimately negate the need to disbud calves and would result in improved calf welfare. However, numerous calves are still disbudded without pain relief (Cozzi et al., 2015; USDA, 2018) and selection for polled cattle has not yet been readily adopted by the industry (Spurlock et al., 2014). Therefore, there remains a need to evaluate novel disbudding techniques or refine current techniques to improve the welfare of calves during disbudding. 
Recently, novel methods of disbudding have been evaluated such as cryoablation (freezing of the horn bud cells with liquid nitrogen; Bengtsson et al., 1996; Stewart et al., 2014) and clove oil (Molaei et al., 2014; Sutherland et al., 2018). Calves spent more time lying $(\sim 1.5 \mathrm{~h} / 24 \mathrm{~h})$ after cryoablation than after cautery disbudding, and lying times were similar between cryoablation and control calves, which may suggest that these calves were experiencing less discomfort (Stewart et al., 2014). In addition, cryoablation appeared to cause less tissue damage and potentially a reduced inflammatory response (unpublished data). Eugenol (the active ingredient of clove oil) has been used in dentistry as a local anesthetic (Markowitz et al., 1992) and works by inhibiting voltage-gated sodium and calcium channels in trigeminal nociceptors (Park et al., 2006, 2009). It is also used as an anesthetic for fish (Iversen et al., 2003; Pounder et al., 2018). In addition to its analgesic properties, eugenol has been shown to have cytotoxic, antioxidative, and anti-inflammatory properties (refer to review by Kamatou et al., 2012). Furthermore, preventing horn growth by injecting clove oil under the horn bud appeared to cause less initial pain and tissue damage than cautery disbudding (Sutherland et al., 2018). As previous studies suggest, cryoablation and clove oil could provide alternatives to cautery disbudding; the next step is to evaluate whether these methods can reliably prevent horn growth. Therefore, the objectives of this study were to (1) evaluate whether cryoablation or the administration of clove oil is as efficacious as cautery disbudding at preventing horn growth, and (2) evaluate whether the efficacy of cautery disbudding is affected by removing or leaving the horn bud tissue intact after disbudding of dairy calves.

These studies were undertaken on farms located in the Waikato region, North Island, New Zealand $\left(37^{\circ} 47^{\prime}\right.$ S, $175^{\circ} 19^{\prime}$ E) during July 2017 (Southern Hemisphere winter). All procedures involving animals were approved by the Ruakura Animal Ethics Committee (Protocol No. 14215) under the New Zealand Animal Welfare Act 1999.

In a pilot study, 10 Friesian and Friesian cross calves ( $\mathrm{n}=5$ females, $\mathrm{n}=5$ males), approximately $3 \mathrm{~d}$ of age $(3.3 \pm 0.48 \mathrm{~d}$ of age, mean $\pm \mathrm{SD})$ and weighing $33.0 \pm 3.57 \mathrm{~kg}$ (mean $\pm \mathrm{SD}$ ), had $0.5 \mathrm{~mL}$ of clove oil administered under each horn bud. A power analysis was carried out to determine the sample size based on a $5 \%$ significance level and $80 \%$ power. The purpose of the pilot study was to evaluate the effect of clove oil administration on gross anatomical changes to the skull and brain. Clove oil (C8392, 83-85\% eugenol, SigmaAldrich, Saint Louis, MO) was injected subcutaneously using an 18-gauge, half-inch needle as described in
Sutherland et al. (2018). The needle was inserted from the side of the horn bud proximal to the midline and directed under the bud toward the base of the outer portion of the ear. Clove oil was injected once the tip of the needle was situated under the center of the horn bud. Calves were initially fed $2 \mathrm{~L}$ of milk/calf twice daily, which increased to 2.5 to $3 \mathrm{~L}$ of milk/calf twice daily; all calves had ad libitum access to meal, lucerne cubes, and water.

Five calves were euthanized at $11 \mathrm{~d}$ of age and 5 at 31 $\mathrm{d}$ of age to evaluate the effect of clove oil administration on the skull and brain as assessed by gross anatomical examination. Calves were euthanized by a veterinarian, and the heads and horn bud sites were examined by a trained veterinary pathologist (New Zealand Veterinary Pathology, Hamilton, New Zealand) to assess exterior tissue damage to the skin and skull tissue (i.e., ulcerations, necrosis, and hemorrhage). The heads were cut transversely using a commercial meat band saw just caudal to the horn buds. The brain was then removed and the intracranial surface of the frontal bone was examined for evidence of injury (i.e., discoloration, indentation) or inflammation beneath the horn bud sites. The dorsal surface of the cerebral hemispheres beneath these sites were examined for ulcerations, discoloration, or any other abnormalities. Clove oil did not cause any damage to the skull or brains of the calves, so we proceeded to conduct an efficacy trial.

At approximately $4 \mathrm{~d}$ of age $(4.1 \pm 0.94 \mathrm{~d}$ of age, mean \pm SD), 271 dairy heifer calves were enrolled on 3 dairy farms. A power analysis for a binary outcome bio-equivalence trial was conducted based on $80 \%$ power and a $5 \%$ significance level, an underlying efficacy rate of $90 \%$, an equivalence range of $\pm 10 \%$, and the assumption that the true efficacy is the same for all treatment groups and all farms. Each calf had 1 of 4 treatments randomly assigned to each horn bud: (1) clove oil $(0.5 \mathrm{~mL})$ administered subcutaneously under the horn bud (CLOV, n = 138 buds); (2) a liquid nitrogen-filled probe applied to the horn bud area for 30 s (CRYO, $\mathrm{n}=135$ buds); (3) cautery disbudded using an electric hot-iron applied for approximately 15 $\mathrm{s}$ and the horn bud tissue removed (BUDOFF, $\mathrm{n}=$ 134); or (4) cautery disbudded using an electric hotiron applied for approximately $15 \mathrm{~s}$ and the horn bud tissue left intact (BUDON, $\mathrm{n}=135$ ). The experiment had the structure of an incomplete block design, and CycDesign4 (Genstat statistical software, version 18, VSN International, Hemel Hempstead, UK) was used to generate the design and randomize the allocation of treatments to animals. Calves were restrained manually in their home pen, the hair within the horn bud area was shaved, and $4 \mathrm{~mL}$ of local anesthetic $(2 \%$ 
lignocaine hydrochloride; Lopain, Ethical Agents Ltd., Auckland, New Zealand) was injected into the cornual nerve. After at least $5 \mathrm{~min}$, calves were moved into and restrained in a sheep crate (Tru Test Ltd., Auckland, New Zealand), which was modified to include a Calf Mate Dehorning Bail head restraint (Farmquip Ltd., Napier, New Zealand). All calves were weighed, and then a clostridial vaccine $(2 \mathrm{~mL}$, Covexin 10 , Schering-Plough Animal Health Limited, Upper Hut, New Zealand) and a nonsteroidal anti-inflammatory drug (Metacam, Norbrook Laboratories Limited, Auckland, New Zealand) were administered s.c. The calf's head was then restrained and the allocated disbudding treatment performed. Clove oil was administered using the same methodology as in the pilot. Cryoablation involved placing a flat cylindrical hollow probe $(24 \mathrm{~mm}$ diameter) onto the horn bud for $30 \mathrm{~s}$, which was cooled continuously by filling the hollow cylinder of the probe with liquid nitrogen using a commercial spray applicator (Cry-Ac, Bry-Mill Cryogenic Systems, Ellington, CT). In the present study, we applied the cryoablation treatment using a cooled probe rather than by spraying liquid nitrogen directly onto the horn bud region, as we believed that this method would be more practical (e.g., less risk of liquid nitrogen getting into the calf's eye). A preliminary histological study suggested that this method caused sufficient necrosis of the horn bud cells (unpublished data). For cautery disbudding, an electric cautery iron ("Quality" electric debudder, 230 V, 190 W; Lister GmbH, Lüdenscheid, Germany) was applied to the horn bud for approximately $15 \mathrm{~s}$; the horn bud was then left intact (BUDON) or removed (BUDOFF). After administration of treatments, calves were returned to the home pen. Figure 1 shows im- ages of the horn bud region approximately 1 wk after CLOV, CRYO, BUDOFF, and BUDON treatments were performed. Calves were reared per normal farm practice for each farm: initially, calves were fed $2 \mathrm{~L}$ of milk/calf twice daily which increased to 2.5 to 3 L milk/calf twice daily, and all calves had ad libitum access to meal and water.

Within the first month after disbudding, calves were assessed frequently for signs of infection (e.g., presence of exudate, pus, or swelling) at the site of the disbudding wound by a veterinarian and a trained technician. At approximately 6 mo $(6 \pm 2.2$ mo, mean $\pm \mathrm{SD})$ after disbudding, calves were assessed for signs of scur or horn growth by the same veterinarian and trained technician. During the 1-mo assessment, it was not possible for the assessors to be blind to the treatments because of the differences in appearance of the treatments; however, assessors were blind to the treatment during the 6-mo assessment because all wounds had healed by this time. A horn was defined as cornified, thickened keratin in the epidermis shaped like a normally developed horn; a scur was defined as an unattached distorted horn. The disbudding procedure was considered successful if there was no evidence of horn or scur growth.

Six calves died ( 4 buds treated with BUDOFF, 4 with BUDON, 1 with CRYO, and 3 with CLOV) during the study period. Calves were necropsied to confirm the cause of death, but no deaths were associated with any of the disbudding treatments. Final numbers of horn buds for each treatment were $\mathrm{n}=135$ buds (CLOV), $\mathrm{n}$ $=134$ buds $(\mathrm{CRYO}), \mathrm{n}=130$ buds $(\mathrm{BUDOFF})$, and $\mathrm{n}$ $=131$ buds (BUDON).

Genstat statistical software (version 18, VSN International) was used to analyze the data. The probability

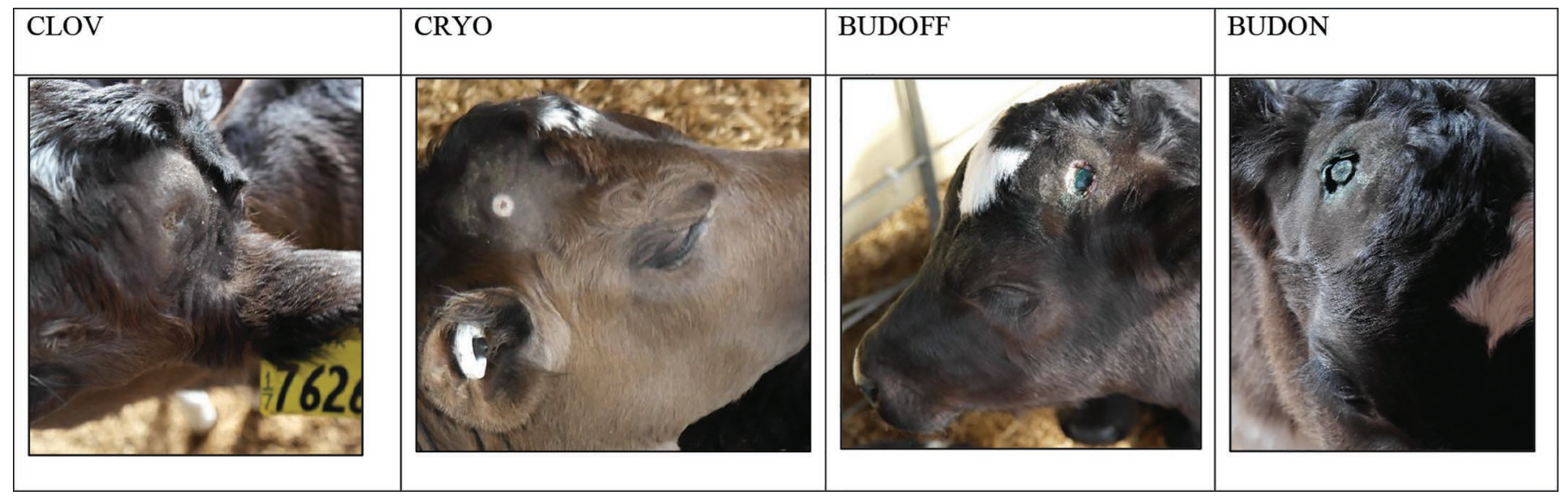

Figure 1. Images of the horn bud region within 1 wk after injecting clove oil subcutaneously under the horn bud (CLOV), applying a liquid nitrogen-cooled probe to the horn bud area (CRYO), using a cautery iron and removing the horn bud tissue (BUDOFF), and using a cautery iron and leaving the bud tissue intact (BUDON). 
of "success" (no evidence of horn or scur growth) was calculated for each treatment (CLOV, CRYO, BUDOFF, and BUDON) along with $95 \%$ confidence intervals (using the exact method). Bio-equivalence of the probability of "success" was assessed between each pair of treatments using a bioequivalence range of \pm 0.10 . That is, the difference between the true values of the probability of success for the 2 treatments had to be within the range of \pm 0.10 to be deemed bioequivalent.

During the first assessment period, $12 \%$ of bud wounds showed some indication of infection such as pus, exudate, or swelling; of the infected buds, $52 \%$ were associated with the BUDON treatment, $27 \%$ with CLOV, $25 \%$ with BUDOFF, and $2 \%$ with CRYO. No sign of infection was observed at the horn bud site at 6 mo of age.

The percentages of buds showing no growth, scurs, or horns for each disbudding treatment are presented in Table 1. At 6 mo, BUDOFF was the most effective method of preventing horn growth, followed by BUDON and CLOV, which prevented horn growth in 100,91 , and $87 \%$ of cases, respectively. The CRYO treatment was ineffective at preventing horn growth; horn growth was prevented in only 1 case. We found no evidence of bio-equivalence for the probability of success between BUDOFF and BUDON or between BUDOFF and CLOV; however, the probabilities of success for BUDON and CLOV were bio-equivalent using a bioequivalence range of $10 \%$. There was no evidence of bio-equivalence for the probability of success between CRYO and any other treatment.

Cautery is the most common method of disbudding dairy calves in New Zealand; hence, in the present study, we wanted to compare the efficacy of cautery with novel methods and different techniques of disbudding. Cauterizing and removing the horn bud was $100 \%$ successful at preventing horn growth in calves in our study. In comparison, injecting clove oil under the horn bud tissue was $87 \%$ successful at preventing any horn growth in the present study. Molaei et al. (2014,
2015) also found that injecting clove oil was effective at preventing horn growth in calves and goat kids; however, they used very small numbers of animals in their studies. The present study confirmed that clove oil can effectively prevent horn growth in calves; however, $13 \%$ of calves grew scurs (but not horns). This could be due to the method of clove oil administration used in our study, which involved injecting the oil with a needle and syringe. It was often difficult to restrain a calf's head, which could have led to inadequate placement of the oil. Hence, sedating the calves before disbudding may have been beneficial. However, before clove oil could be considered a potential alternative to cautery disbudding, the method of administration needs to be refined and the efficacy of this procedure would need to be bioequivalent to BUDOFF at preventing horn growth. In addition, it would be of interest to observe animals at 12 mo of age to confirm that clove oil actually does prevent horn growth and not just delay horn development.

In our pilot study, injecting clove oil subcutaneously under the horn bud did not cause any damage to the skull or brain of calves. In addition, Sutherland et al. (2018) reported that injecting clove oil under the horn bud appeared to be less painful initially, and did not appear to cause more pain than cautery disbudding in the $48 \mathrm{~h}$ after treatment. Therefore, using clove oil to prevent horn growth in calves does not appear to cause more pain and could cause fewer acute or long-term welfare concerns than cautery disbudding. However, some swelling of the horn bud region was observed after injecting clove oil; therefore, there is a need to further understand the effects of clove oil on tissue damage and the welfare of calves. In particular, it is necessary to know the mechanisms of action of clove oil on the horn bud and how calves perceive it so that appropriate pain mitigation can be provided if necessary.

Cryoablation prevented horn growth in only one case. This may be due to the method of using a probe in the present study, as Stewart et al. (2014) reported

Table 1. Mean percentage (with 95\% CI) of buds with no horn growth, scurs, or horns 6 mo after calves were disbudded using different methods ${ }^{1}$

Disbudding method ${ }^{1}$

\begin{tabular}{|c|c|c|c|c|c|c|c|c|}
\hline Outcome & \multicolumn{2}{|c|}{ BUDOFF } & \multicolumn{2}{|c|}{ BUDON } & \multicolumn{2}{|c|}{ CLOV } & \multicolumn{2}{|c|}{ CRYO } \\
\hline Scurs & 0 & $0.0-2.3$ & 8 & $4.3-14.5$ & 13 & $8.1-18.5$ & 22 & $15.6-30.4$ \\
\hline Horns & 0 & $0.0-2.3$ & 1 & $0.0-4.2$ & 0 & $0.0-2.2$ & 76 & $68.0-83.1$ \\
\hline
\end{tabular}

${ }^{1}$ BUDOFF $=$ using a cautery iron and removing the horn bud tissue removed $(\mathrm{n}=130)$; BUDON $=$ using a cautery iron and leaving the bud tissue intact $(\mathrm{n}=131)$; CLOV = injecting clove oil subcutaneously under the horn bud $(\mathrm{n}=135$ buds); and CRYO = applying a liquid nitrogen-cooled probe to the horn bud area ( $\mathrm{n}=134$ buds). 
that spraying liquid nitrogen directly onto the horn bud site had a 47 and $30 \%$ success rate at preventing horn growth when applied for 15 or $10 \mathrm{~s}$, respectively. Bengtsson et al. (1996) also found that applying liquid nitrogen using a cryoprobe was less efficacious at preventing horn development in calves than applying liquid nitrogen directly onto the skin. However, we used the probe technique in the present study because we thought that it may be a more practical method of application. Because of the potential of cryoablation to prevent horn growth (using other methodologies), while simultaneously causing less tissue damage, this technique has potential as a more humane alternative to cautery disbudding but further refinement of the technique, including the timing, is required. In addition to causing less pain than the current established methods of disbudding, cryoablation would need to be bioequivalent to BUDOFF at preventing horn growth before it could be considered an acceptable alternative method of disbudding.

It is common to remove the cauterized horn bud tissue after cautery disbudding in calves. This is done to prevent horn growth due to remaining viable germinal epithelial cells; however, removing the horn bud leaves calves with an exposed open wound. In the present study, we wanted to evaluate whether leaving the horn bud tissue intact after cautery disbudding would affect the efficacy of this procedure and wound healing. Interestingly, leaving the horn bud tissue intact reduced the efficacy of cautery disbudding by $9 \%$ and increased the amount of infection observed. Efficacy may have been reduced because viable germinal epithelium was still present, which was likely the result of an insufficient cut or incomplete burn made by the cautery iron, resulting in the formation of scurs. Moreover, the increased risk of infection was likely due to the intact horn bud tissue becoming necrotic. These results suggest that removing the horn bud tissue is preferable to leaving it intact after cautery disbudding.

In conclusion, it appears advantageous to remove the horn bud tissue after cautery disbudding to increase efficacy and prevent possible infections caused by this procedure. Cautery disbudding (horn bud removed) was $100 \%$ successful at preventing horn growth; however, liquid nitrogen applied for $30 \mathrm{~s}$ using a probe was ineffective. However, it appears as though clove oil could be used as an alternative to cautery disbudding in dairy calves, although further research is needed to evaluate the tissue damage and associated pain caused by clove oil and to refine this technique (i.e., administration methods to improve efficacy). Furthermore, several barriers would currently prevent adoption of this method by the industry that would first need to be overcome, such as age of disbudding, potential drug residues, and drug regulatory issues. Moreover, because disbudding with clove oil appears to cause calves stress, it is recommended that calves be provided with pain relief before using this method.

\section{ACKNOWLEDGMENTS}

The authors thank AgResearch staff, in particular Gemma Lowe, Ali Cullum, and Rosalie Carter. Also, Johan Larive, Benjamin Ferviot, and participating farmers. AgResearch (Hamilton, New Zealand) also gratefully acknowledges this funding by the New Zealand Ministry of Business, Innovation and Employment.

\section{REFERENCES}

Bengtsson, B., A. Menzel, P. Holtenius, and S. O. Jacobsson. 1996. Cryosurgical dehorning of calves: A preliminary study. Vet. Rec. 138:234-237.

Cozzi, G., F. Gottardo, M. Brscic, B. Contiero, N. Irrgang, U. Knierim, O. Pentelescu, J. J. Windig, L. Mirabito, F. Kling Eveillard, A. C. Dockes, I. Veissier, A. Velarde, C. Fuentes, A. Dalmau, and C. Winckler. 2015. Dehorning of cattle in the EU Member States: A quantitative survey of the current practices. Livest. Sci. 179:4-11.

Iversen, M., B. Finstad, R. McKinley, and R. Eliassen. 2003. The efficacy of metomidate, clove oil, Aqui-S ${ }^{\mathrm{TM}}$ and Benzoak ${ }^{\circledR}$ as anaesthetics in Atlantic salmon (Salmo salar L.) smolts, and their potential stress-reducing capacity. Aquaculture 221:549-566.

Kamatou, G. P., I. Vermaak, and A. M. Viljoen. 2012. Eugenol-From the remote Maluku Islands to the international market place: A review of a remarkable and versatile molecule. Molecules 17:69536981.

Markowitz, K., M. Moynihan, M. Liu, and S. Kim. 1992. Biologic properties of eugenol and zinc oxide-eugenol: A clinically oriented review. Oral Surg. Oral Med. Oral Pathol. 73:729-737.

McMeekan, C. M., K. J. Stafford, D. J. Mellor, R. A. Bruce, R. N. Ward, and N. Gregory. 1998. Effects of regional analgesia and/or a non-steroidal anti-inflammatory analgesic on the acute cortisol response to dehorning in calves. Res. Vet. Sci. 64:147-150.

Molaei, M. M., O. Azari, and S. Esmaeilzadeh. 2014. Study of calves disbudding following injection of clove oil under horn bud. J. Vet. Res. (Pulawy) 69:363-369.

Molaei, M. M., A. Mostafavi, R. Kheirandish, O. Azari, and M. Shaddel. 2015. Study of disbudding goat kids following injection of clove oil essence in horn bud region. Vet. Res. Forum 6:17-22.

Morisse, J. P., J. P. Cotte, and D. Huonnic. 1995. Effect of dehorning on behavior and plasma-cortisol responses in young calves. Appl. Anim. Behav. Sci. 43:239-247.

Park, C.-K., K. Kim, S. J. Jung, M. J. Kim, D. K. Ahn, S.-D. Hong, J. S. Kim, and S. B. Oh. 2009. Molecular mechanism for local anesthetic action of eugenol in the rat trigeminal system. Pain 144:84-94.

Park, C.-K., H. Y. Li, K.-Y. Yeon, S. J. Jung, S.-Y. Choi, S. J. Lee, S. Lee, K. Park, J. S. Kim, and S. B. Oh. 2006. Eugenol inhibits sodium currents in dental afferent neurons. J. Dent. Res. 85:900-904.

Pounder, C., J. Mitchell, J. Thomson, T. Pottinger, and L. Sneddon. 2018. Physiological and behavioural evaluation of common anaesthesia practices in the rainbow trout. Appl. Anim. Behav. Sci. 199:94-102.

Spurlock, D. M., M. L. Stock, and J. F. Coetzee. 2014. The impact of 3 strategies for incorporating polled genetics into a dairy cattle breeding program on the overall herd genetic merit. J. Dairy Sci. 97:5265-5274. 
Stafford, K. J., and D. J. Mellor. 2011. Addressing the pain associated with disbudding and dehorning in cattle. Appl. Anim. Behav. Sci. 135:226-231.

Stewart, M., S. Dowling, K. Schultz, V. Cave, and M. Sutherland. 2014. Liquid nitrogen cryosurgery as an alternative to hot-iron disbudding in dairy calves. Page 217 in 48th Congr. Int. Soc. Appl. Ethol., Vitoria-Gasteiz, Spain. Wageningen Academic Publishers, Wageningen, the Netherlands.

Stilwell, G., R. C. de Carvalho, M. S. Lima, and D. M. Broom. 2009. Effect of caustic paste disbudding, using local anaesthesia with and without analgesia, on behaviour and cortisol of calves. Appl. Anim. Behav. Sci. 116:35-44.

Sutherland, M. A., J. Larive, V. Cave, and G. Zobel. 2018. Behavioural and physiological responses to clove oil injected under the horn bud of calves. Appl. Anim. Behav. Sci. 204:29-36.
Sylvester, S. P., K. J. Stafford, D. J. Mellor, R. A. Bruce, and R. N. Ward. 2004. The behavioural responses of calves to amputation dehorning with and without local anaesthesia. Aust. Vet. J. $82: 697-700$.

USDA. 2018. Dairy 2014, Report 3: Health and Management Practices on U.S. Dairy Operations, 2014. No. 696.0218. USDA-Animal and Plant Health Inspection Service (APHIS)-Veterinary Services (VS)-Center for Epidemiology and Animal Health (CEAH)-National Animal Health Monitoring System (NAHMS), Fort Collins, CO. Accessed Mar. 1, 2018. https://www.aphis.usda.gov/animal _health/nahms/dairy/downloads/dairy14/Dairy14_dr_PartIII .pdf. 